\title{
Sensitivity and Performances Analysis of a Dynamic Pressure Narrow-Band Electrodynamic Micro- Sensor
}

\author{
Mohamed Hadj Said \\ METS Research Group, National Engineering School of Sfax, University of Sfax, Route Soukra, BP 1173, 3038 \\ Sfax, Tunisia.
}

Fares Tounsi

METS Research Group, National Engineering School of Sfax, University of Sfax, Route Soukra, BP 1173, 3038 Sfax, Tunisia.

Sensors, Microsystems and Actuators Laboratory of Louvain (SMALL), Université Catholique de Louvain, Place du Levant, 3, 1348 Louvain-la-Neuve, Belgium.

\section{Libor Rufer}

University Grenoble Alpes, CNRS, Grenoble INP, TIMA, 38000 Grenoble, France.

\section{Brahim Mezghani}

METS Research Group, National Engineering School of Sfax, University of Sfax, Route Soukra, BP 1173, 3038 Sfax, Tunisia.

\section{Laurent A. Francis}

Sensors, Microsystems and Actuators Laboratory of Louvain (SMALL), Université Catholique de Louvain, Place du Levant, 3, 1348 Louvain-la-Neuve, Belgium.

(Received 27 February 2018; accepted 6 June 2018)

This paper presents analytic and numerical modelling of a MEMS electrodynamic micro-sensor of dynamic pressure. Two coaxial planar inductors of different diameters are used in the proposed micro-sensor design. Using finite element analysis, the diaphragm resonant frequency and dynamic displacements are evaluated for different diaphragm thicknesses. Then, the total sensitivity is deduced by coupling different physical domains which contribute in the micro-sensor operation. A lumped element model is built in order to study the micro-sensor sensitivity and define the dynamic performance for different resonant frequencies. This model shows that the best sensitivity, within the $\mathrm{mV} / \mathrm{Pa}$ range, is obtained around the resonant frequency when operation in the audible frequency range, and decreases to the $\mu \mathrm{V} / \mathrm{Pa}$ range for ultrasonic frequencies. The obtained sensitivity curves prove that the undamped inductive micro-sensor can offer high pressure sensitivity within a narrow frequency bandwidth.

\section{INTRODUCTION}

Micro-electro-mechanical systems, or MEMS, are generally considered as micro devices consisting of a combination of micro mechanical sensors and/or actuators in addition to microelectronic circuits. ${ }^{1}$ For the last several decades, MEMS-based sensors have been under steady development, and a variety of devices have been manufactured aiming numerous applications like accelerometers, ${ }^{2}$ gyroscopes, ${ }^{3}$ magnetometers, ${ }^{4}$ and acoustic applications. ${ }^{5,6}$ Acoustic microsystem devices have attracted the attention of several research groups, in order to achieve various frequency responses and bandwidths. The main objectives have been the miniaturization of existing acoustic macro-devices to microscopic scale in order to reduce costs, and to obtain acoustic sensing de- vices with improved characteristics (dimensions, frequency range, signal/noise ratio, etc.). These characteristics are difficult to achieve with traditional fabrication processes given the limited resolution and high tolerance on the geometrical dimensions. ${ }^{5}$ MEMS-based acoustic devices can be divided into two main areas: audible frequency sensors/actuators, such as microphones and micro-speakers sensing or generating an airborne acoustic signal; ${ }^{7,8}$ and ultrasound sensors/actuators such as filters and resonators devices based on surface acoustic waves (SAW) or bulk acoustic waves (BAW) using an acoustic signal propagated in solid state. ${ }^{9,10}$ Microphones are a kind of pressure sensor designed to convert an acoustic signal of audible frequency into an electrical signal, differently from micro-speakers, which have an opposite role. The conversion effects mainly used are piezoelectricity, piezoresistivity, 
electrostatic, and electromagnetic methods. ${ }^{8,11-13}$ The electrostatic transduction is the most common scheme used in silicon microphones because of its high sensitivity $(\sim \mathrm{mV} / \mathrm{Pa})$, large bandwidth and low noise level. Piezoresistive transducers are robust due to their simple structure, nevertheless they show a low sensitivity and the piezoresistive material can suffer from thermal degradation due to the Joule heating effect. Finally, the piezoelectric method is very common for high frequency applications of narrow bandwidth. Electromagnetic conversion is based on the electrodynamic theory and is used in traditional microphones and speakers. Electrodynamic microsensors offer low self-noise and low internal impedance and have no preamplifier, so external power supplies are not required. ${ }^{14}$ Although, the basic principle of miniaturized inductive transducers using the interaction between a coil and the electromagnetic field does not change; there are numerous ways of their design and miniaturization. A MEMS-based magnetic micro-speaker with polyimide flat membrane having the size of $2.5 \mathrm{~mm} \times 2.5 \mathrm{~mm}$ and the thickness of $2 \mu \mathrm{m}$ was detailed in Sugandi and Majlis. ${ }^{8}$ This device was fabricated by bonding two silicon substrates, the upper wafer supporting a coil mounted on a polyimide membrane, and the lower wafer holding a sandwiched permanent magnet disc of NdFeB. Similarly, a MEMS-based dual-planar-coil miniature dynamic micro-sensor was described in Horng et al. ${ }^{14}$ It consisted of a $1 \mu \mathrm{m}$ thick diaphragm sandwiched between two spiral coils and vibrating in the region where the highest magnetic flux density was generated by a double magnetic system. In Bakhoum, the electrodynamic transduction was based on the creation of a highly variable inductance mechanism caused by the relative magnetic permeability variation of the corematerial. ${ }^{15}$ So, a cylindrical iron core of a height of $4 \mathrm{~mm}$ and a diameter of $6 \mathrm{~mm}$ was moving inside a 24-pin DIP embedded coil, under the effect of the sound pressure. The same principle was implemented in Arshad and Tasnim, where a diaphragm moving in front of an inductive coil caused significant variations of its inductance. ${ }^{16}$ All these attempts were either combining a diaphragm built in a MEMS technology combined with a macro-magnet core embedded in an external package, or they were made in a cm-range size. Our design was original compared to other inductive transducers, since the B-field was generated by an outer inductor, and so the inductance will vary due to the variation of the mutual inductance between the two inductors. The proposed solution, compared to other devices, has the advantage to be CMOS-compatible with a single etching post-process step. The monolithic integration will increase performance, miniaturize the system, and in particular, improve noise reduction, due to the reduction of interconnection parasitic capacitances. ${ }^{17}$ The objective of the current study was to determine the influence of geometric parameters on the performances of the electrodynamic transduction in resonant devices for different acoustic applications. Such narrow-band devices can be used in various tasks. One application example can be the detection of acoustic signals generated by partial discharge from high electric voltage cables. The frequency range of these signals was around $40 \mathrm{kHz}$, and was well matching the design window of the proposed sensor (such as the Ul-

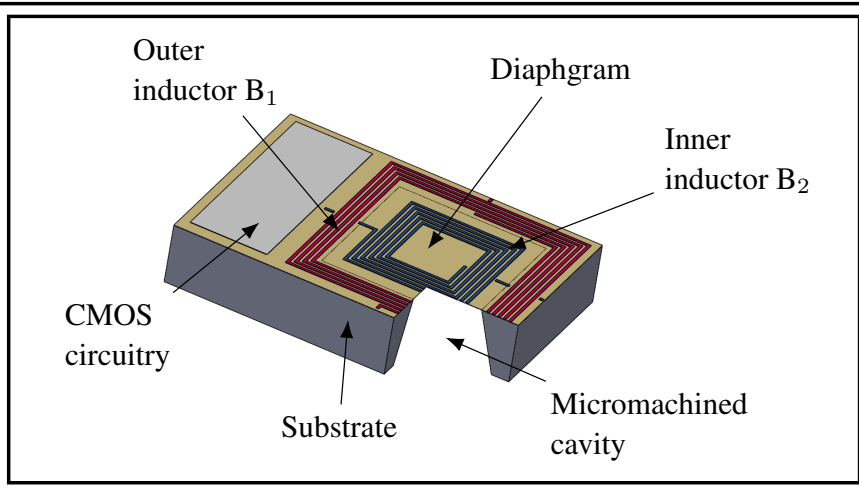

Figure 1. 3-D representation of the electrodynamic micro-sensor structure.

trasonic PD Sensor LDA-5/S). ${ }^{18}$

The paper is organized as follows: after the operating principle description of the micro-sensor, we will detail a mechanical modeling of the micromachined vibrating part using both analytical and FEM analysis for different diaphragm thicknesses. The modeling will itemize the diaphragm dimensions, which offers multiple targeted dynamic performances in accordance with the intended application. In the last section, the overall sensitivity will be evaluated by finding the coupling schemes between the involved transduction domains (acoustic-mechanical-electromagnetic), and the characteristics of each part of the sensor.

\section{MICRO-SENSOR OPERATING PRINCIPLE}

The electrodynamic micro-sensor (called also inductive, magneto-dynamic or dynamic) is very common in its traditional form having the size of units to tens of millimetres, but can be considered as emerging when using MEMS technology. The proposed structure consisted of two coaxial, concentric planar squared inductors (see Fig. 1); a fixed outer inductor $B_{1}$ was placed on the substrate top surface and an inner inductor $\mathrm{B}_{2}$ was implemented on a squared diaphragm suspended over a micromachined cavity. The diaphragm consisted of a superposition of several layers, which were mainly silicon dioxide and silicon nitride. ${ }^{19}$ When a DC bias current flowed in $\mathrm{B}_{1}$, this led to the generation of a time-independent permanent magnetic field within $B_{2}$. By referring to Faraday's law of induction, any change in the magnetic flux magnitude through a closed loop wire generated an induced voltage, also called an electromotive force, emf. So, the vertical fluctuation of the inner inductance $\mathrm{B}_{2}$ (embedded to the diaphragm), due to the incident acoustic waves within the created magnetic field, generated, at its ends, an induced output voltage proportional to the displacement amplitude. In a previous work, the inductor's size, section, and spacing were optimized to enhance the produced magnetic field. ${ }^{20}$

\section{MODELLING OF THE ELECTRODYNAMIC SENSOR}

\subsection{Diaphragm Static Displacement}

The micro-sensor diaphragm was designed to be attached through its periphery to the substrate. To avoid the use of open- 
ings around the diaphragm, a back-side bulk micromachining technique was used to suspend the structure. ${ }^{21}$ This was used in order to eliminate possible acoustic short paths toward the low frequencies, between the ambient air, and the cavity underneath the diaphragm in order to widen the micro-sensor bandwidth. ${ }^{22,23}$

To describe the normal movement of a clamped squared plate under the action of a sound pressure $P_{i n}$, the following expression was used: ${ }^{22}$

$$
D\left(\frac{\partial^{4} w(x, y)}{\partial x^{4}}+2 \frac{\partial^{4} w(x, y)}{\partial x^{2} \partial y^{2}}+\frac{\partial^{4} w(x, y)}{\partial y^{4}}\right)=P_{i n}
$$

where $w$ was the normal displacement of the point with coordinates $(x, y)$ situated on the diaphragm top surface, and $D$ was the flexural rigidity of the plate, given by:

$$
D=\frac{E_{a v g} t_{h}^{3}}{12\left(1-v^{2}\right)}
$$

where $E_{\text {avg }}$ was the Young's modulus of the equivalent stacked materials (to be determined later), and $v$ was its equivalent Poisson's ratio. The diaphragm thickness $t_{h}$ was a technological parameter to be fixed during the fabrication process. Since the diaphragm structure was implemented using different materials, adjustments had to be introduced to Eq. (2). Specifically, the average mechanical proprieties, such as Young's modulus and density, should be accounted for, and this adjustment led to: ${ }^{24}$

$$
E_{\text {avg }}=\frac{\sum_{i=1}^{n} E_{i} t_{i}}{\sum_{i=1}^{n} t_{i}} \text { and } \rho_{\text {avg }}=\frac{\sum_{i=1}^{n} \rho_{i} t_{i}}{\sum_{i=1}^{n} t_{i}}
$$

where $n$ was the number of used diaphragm layers and the index $i$ reflected the specific layer number forming the diaphragm. As a result, the maximum displacement of a squared diaphragm, under a static applied pressure $P_{i n}$, was located in the centre, and was expressed by: ${ }^{1}$

$$
\delta_{\text {static }}=P_{\text {in }} \frac{L^{4}}{1184 D}
$$

Figure 2 plots both the FEM and analytic static displacement values of the diaphragm center for different applied incident pressure magnitudes. The slight deviation of values obtained from the FEM and analytic studies were explained by the fact that in FEM simulation, the secondary effect was accounted for, as opposed to the analytic study where many side effects were neglected. The deviation of values obtained from FEM and analytic studies could be explained by the facts that $E_{\text {avg }}$ and $t_{\text {avg }}$ considered in Eq. (3), were slightly different from the ones computed by $\mathrm{Comsol}^{\circledR}$. In addition, due to the high diaphragm aspect ratio, the discretization meshes were not as regular as required. As a result, the mesh parallelepipeds had a stretched geometry, which affected the accuracy. A use of correct parallelepipeds could increase the mesh density, and therefore increased the simulation time unnecessarily. This deviation could certainly be accepted as long as the difference did not exceed $10 \%$, which was largely valid in our case.

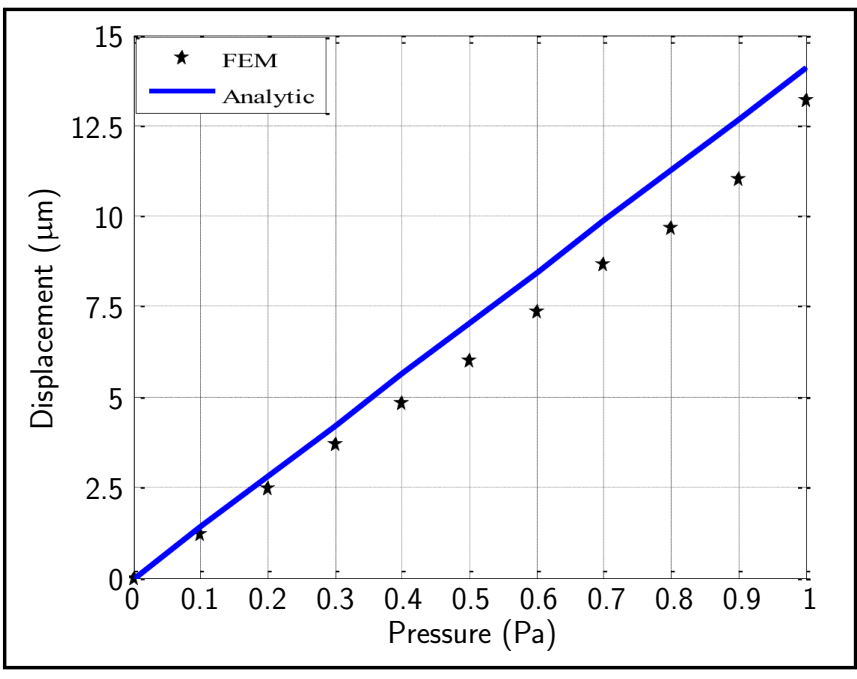

Figure 2. FEM and analytic calculation of the static displacement of the diaphragm center as a function of incident pressure.

Table 1. Eigenmode coefficients, for the first vibration modes of a clamped squared diaphragm without axial stress. ${ }^{26}$

\begin{tabular}{|c|c|c|c|c|}
\hline Mode & $\beta_{1}$ & $\beta_{2}$ & $\beta_{2,2}$ & $\beta_{3}$ \\
\hline Eigenmode coefficient & 5.9992 & 8.5688 & 8.5688 & 10.411 \\
\hline
\end{tabular}

\subsection{Diphragm Resonant Frequencies and Elasticity}

The key parameter of the micro-sensor design was the resonant frequency of the suspended diaphragm, which generally defined the targeted frequency that could be either in the audible or ultrasonic range. The resonant frequency of the suspended structure was fixed by its geometrical parameters such as shape and dimensions, as well as by the type of attachments. This latter should be well defined in order to get an accurate behaviour of the device in the harmonic mode. Generally, the diaphragm structure, attached via a mechanical spring, had an angular frequency of the first natural mode $\omega_{0}=\sqrt{(k / m)}$. Therefore, the relationship between the vibration amplitude and the system frequency had its peak at $\omega_{0}$. The first mode frequency needed to be adjusted according to the targeted sensitivity of the micro-sensor depending on the intended application. Accordingly, for a squared clamped diaphragm, the vibration mode frequencies were defined by: $:^{25,26}$

$$
f_{m}=\frac{\beta_{m}^{2}}{2 \pi L^{2}} \sqrt{\frac{D}{\rho_{a v g} t_{h}}}
$$

where $L$ was the diaphragm side length, $m$ denoted the mode, and $\beta_{m}$ was the corresponding natural frequency coefficient given in Table 1.

As a next step, using various diaphragm thicknesses, we plotted in Fig. 3 its corresponding first mode resonant frequencies as a function of its length, $L$. To be able to clearly compare the results, we used three different diaphragm thicknesses, which led to three resonant frequencies: the first was inside the audible band ( $20 \mathrm{~Hz}$ to $20 \mathrm{kHz}$ ), the second was at the upper limit of the audible band and the third resonant frequency was at the lower limit of the ultrasound range. Using Eq. (5) with a diaphragm length of $1.5 \mathrm{~mm}$, resonant frequency values were 
Table 2. First mode resonant frequency values for selected diaphragm thicknesses.

\begin{tabular}{|c|c|}
\hline Diaphragm thickness $[\mu \mathrm{m}]$ & Resonant frequency $[\mathrm{kHz}]$ \\
\hline 0.3 & 1.619 \\
\hline 3 & 17.48 \\
\hline 10 & 46.28 \\
\hline
\end{tabular}

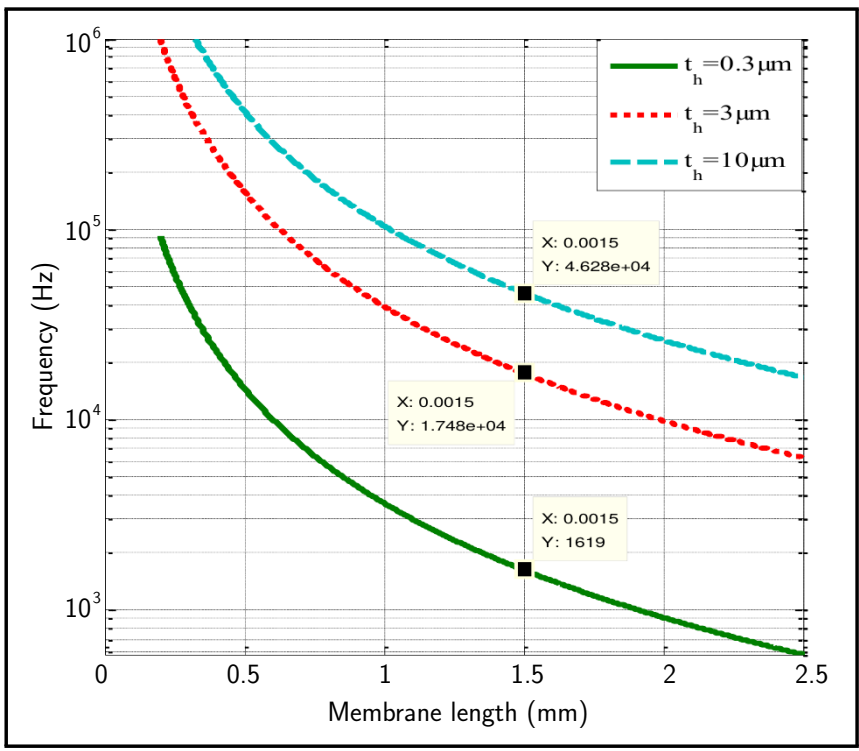

Figure 3. Evaluation of the analytical diaphragm resonant frequency as a function of its side length, $\mathrm{L}$ and thickness, $t_{h}$.

evaluated for the selected thicknesses. Results are summarized in Table 2.

Moreover, Fig. 3 shows that to attain the same resonant frequency, the diaphragm thickness could be increased, or its length could be decreased; however, the latter solution reduced the B-field produced by the outer inductance. For the considered squared diaphragm, the mechanical elastic constant was determined by calculating the maximum displacement for the case of static loading, taking as the basis the first 25 natural modes, without considering axial stress. Based on the numerical evaluation, the calculated spring constant was given by: ${ }^{26}$

$$
K_{\text {dia }}^{m e c}=787.402 \frac{D}{L^{2}}
$$

The effective mass could be immediately calculated from the well-known given relation in Eq. (7), and in the case of squared diaphragm, this yielded to: ${ }^{26}$

$$
M_{d i a}^{m e c}=\frac{K_{d i a}^{m e c}}{\omega_{0}^{2}}=0.607904 \rho t_{h} L^{2} .
$$

To verify the analytic resonant frequency, a modal analysis was performed using the "Solid mechanics" module under $\mathrm{Comsol}^{\circledR}$ Multiphysics software. The boundary conditions applied were fixed by the constraint near the diaphragm edge. The stationary simulation was done using a diaphragm with a side length of $1500 \mu \mathrm{m}$ and thickness of $0.3 \mu \mathrm{m}(0.2 \mu \mathrm{m}$ of silicon dioxide and $0.1 \mu \mathrm{m}$ of silicon nitride). The total mass was evaluated in simulation through the sum of effective mass in each mode using: ${ }^{27}$
Table 3. Comparison of the analytic and FEM simulation of mechanical properties of the squared diaphragm with $1500 \mu \mathrm{m}$ side length and $0.3 \mu \mathrm{m}$ thickness.

\begin{tabular}{||c|c|c|c|}
\hline Diaphragm properties & Analytic & FEM & Difference $(\%)$ \\
\hline $\begin{array}{c}\text { Resonance frequency } \\
\left(f_{r}\right)[\mathrm{kHz}]\end{array}$ & 1.619 & 1.632 & 0.80 \\
\hline $\begin{array}{c}\text { Effective mechanical mass } \\
\left(M_{\text {dia }}\right)[\mu \mathrm{g}]\end{array}$ & 1.025 & 1.048 & 2.19 \\
\hline $\begin{array}{c}\text { Mechanical spring constant } \\
\left(K_{\text {dia }}\right)[\mathrm{N} / \mathrm{m}]\end{array}$ & 0.106 & 0.110 & 3.63 \\
\hline
\end{tabular}

Table 4. Vibration modes evaluation of the squared diaphragm with $1500 \mu \mathrm{m}$ side length and $0.3 \mu \mathrm{m}$ thickness.

\begin{tabular}{||c|c|c|}
\hline Mode & Analytic Result $[\mathrm{kHz}]$ & FEM Result $[\mathrm{kHz}]$ \\
\hline Mode 2,1 & 3.30 & 3.46 \\
\hline Mode 2,2 & 3.30 & 3.47 \\
\hline Mode 3 & 4.87 & 5.13 \\
\hline
\end{tabular}

$$
M_{d i a}^{m e c}=\sum_{i=1}^{N} m_{e f f}(i) ;
$$

where $N$ was the number of evaluated mode (in our case it was calculated for the 6 first modes). The effective mass for each vibration mode, $m_{e f f}(i)$ was calculated through the square of the 'mass participation factor' evaluation in every displacement direction $(x, y, z)$ using $\mathrm{Comsol}^{\circledR}$. Concerning the stiffness, we evaluated the latter by $M_{d i a}^{m e c} . \omega_{0}^{2}$, when using the effective total mass and the resonant frequency, was obtained from simulation. Both simulation and analytic values of the diaphragm properties are summarized in Table 3. We noticed that the differences between both results were slightly different due to meshing sizing. The three next vibration modes of the clamped diaphragm were evaluated using Eq. (5) and FEM analysis, and were gathered in Table 4.

\subsection{Dynamic Displacement Behaviour of the Diaphragm}

Under dynamic excitation with the time dependent applied pressure, the diaphragm displacement was strongly dependent on the frequency of the incident pressure wave. Then, for frequencies below the first natural frequency (and around it), suspended structures can be modelled using a second order mechanical system represented by a mass with a damping spring. This linear model gave good results as the fluctuation had small amplitudes. The dynamic response of such a system corresponded to the solution of the following differential equation:

$$
M \frac{d^{2} x}{d t^{2}}+b \frac{d x}{d t}+K x=f_{e x t}
$$

where $M$ was the effective mass of the suspended diaphragm, $K$ was the spring constant of the attachment structure, $b$ was the damping coefficient (equivalent to mechanical resistance), $x$ was the mass displacement and $f_{\text {ext }}$ was the sum of the external forces applied to the structure (corresponding to external sound pressure in the microphone case). Using the Laplace transform, the frequency response could be expressed as: 


$$
X(j \omega)=\frac{F_{e x t}(j \omega)}{k}\left(\frac{1}{1-\left(\omega / \omega_{0}\right)^{2}+j \omega / Q \omega_{0}}\right)
$$

where $\omega_{0}=\sqrt{(k / m)}$ was the resonant angular frequency and $Q=k /\left(\omega_{0} b\right)$ defined the quality factor of the vibrating structure. The term $\xi=b /(2 \sqrt{(\mathrm{km})})$ was defined as the damping ratio of the system. On this basis, a harmonic simulation was performed using the "Shell" module under Comsol ${ }^{\circledR}$ Multiphysics to obtain the dynamic diaphragm displacement. By applying a sine pressure of amplitude $0.1 \mathrm{~Pa}$, and under a damping ratio equal to 0.1 ; meant that $10 \%$ of the critical damping which was given by $2 \sqrt{(\mathrm{km})}$. The frequency response of the plate was obtained using a frequency sweep in the resonance neighborhood of the obtained analytic values found in Table 2. In our study, we assumed the simplest case, in which the acoustic wave was purely sinusoidal with fixed amplitude. FEM simulations of the displacement in the diaphragm center as a function of the frequency were plotted in Fig. 4 and were confirmed theoretically using Eq. (10). A slight difference existed between the simulation and analytic model results due to the use of a second order suspended model approximation in the mechanical modeling. We could note that the maximal displacement was found around the previously evaluated resonant frequencies (which are $1.6 \mathrm{kHz}, 17.5 \mathrm{kHz}$ and $46.3 \mathrm{kHz}$ ). The diaphragm center displacement decreased when increasing the diaphragm thickness, which induced higher resonant frequency (Fig. 3). This result was expected since the displacement diminished with increasing diaphragm stiffness.

\section{SENSITIVITY EVALUATION OF THE ELECTRODYNAMIC MICRO-SENSOR}

\subsection{Basis of the Used Electro-Acoustic Modelling}

To be able to estimate the micro-sensor sensitivity, we needed to extract an equivalent lumped element model imitating the physical device behaviour. The lumped model must gather mechanical, acoustic, and electromagnetic phenomena present in the system. To this end, an analogy between different involved physical domains must be used. This eased the solution of mechanical and/or acoustic vibrating systems through converting these coupled problems to the corresponding electrical domain and then solving the resulting electrical circuit using classical theory. The acoustic elements encountered in the equivalent model involve resistance, mass, or compliance (see Table 5).

When applying analogy between the different energy fields, a lumped element model of the micro-sensor could be deduced. This analogy consisted in connecting in series all elements crossed by the same acoustic flow and in parallel elements corresponding to a flow addition. From the lumped element modelling, we determined the sensitivity, and the frequency response (in particular amplitude and phase); the complex acoustic impedance of the diaphragm, the electrical impedance and the intrinsic electrical noise of the micro-sensor. In the next

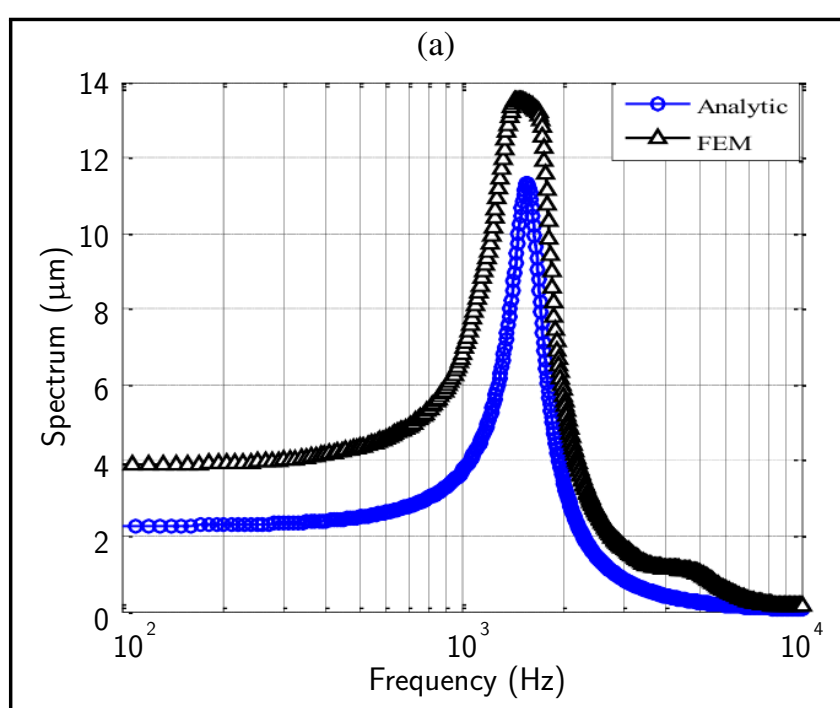

(b)

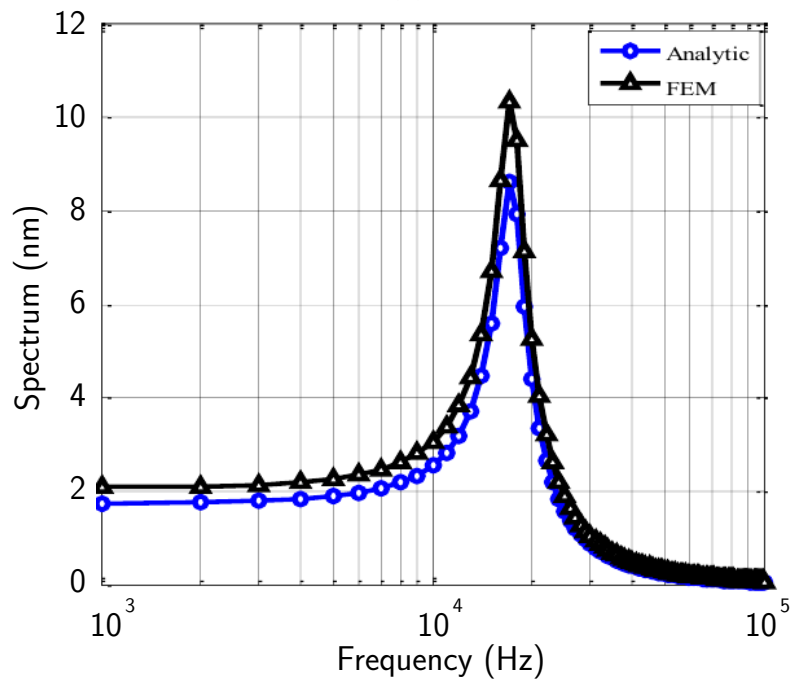

(c)

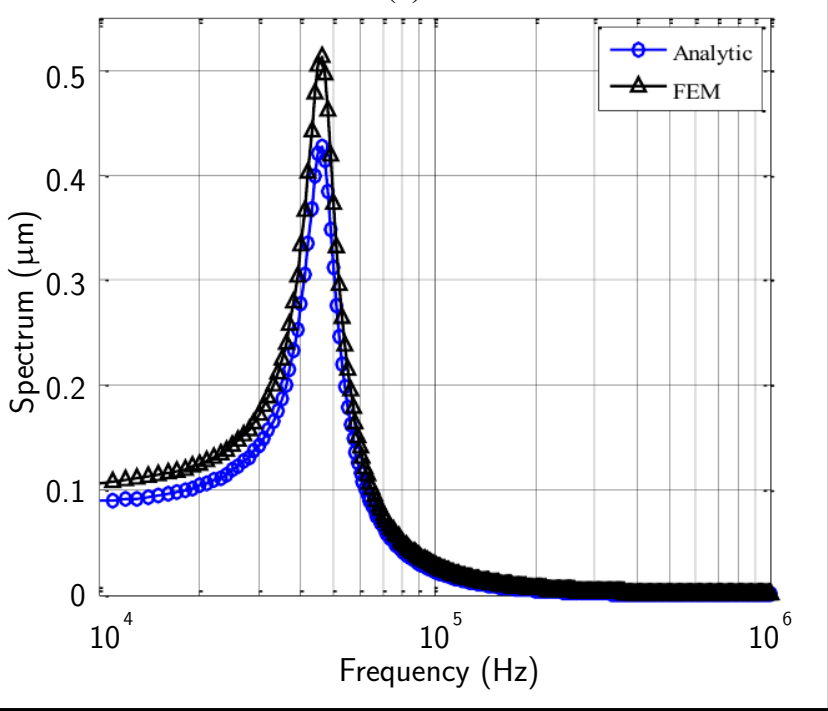

Figure 4. Diaphragm center displacement as a function of the frequency, for a diaphragm length of $1500 \mu \mathrm{m}$ and thickness equal to (a) $t_{h}=0.3 \mu \mathrm{m}$, (b) $t_{h}=3 \mu \mathrm{m}$, (c) $t_{h}=10 \mu \mathrm{m}$. 
Table 5. Analogy conversion table between the acoustic, electrical, and mechanical domains.

\begin{tabular}{||c|c|c|c|c|c||}
\hline Acoustic Parameter & Unit & $\begin{array}{c}\text { Electrical } \\
\text { equivalent parameter }\end{array}$ & Unit & $\begin{array}{c}\text { Mechanical } \\
\text { equivalent parameter }\end{array}$ & Unit \\
\hline Pressure $p$ & $\mathrm{~Pa}=\mathrm{N} / \mathrm{m}^{2}$ & Voltage, $U$ & $\mathrm{~V}$ & Force, $F$ & $\mathrm{~N}$ \\
\hline Flow rate $w$ & $\mathrm{~m}^{3} / \mathrm{s}$ & Current, $I$ & A & Velocity, $v$ & $\mathrm{~m} / \mathrm{s}$ \\
\hline Volume displacement, $z$ & $\mathrm{~m}^{3}$ & Charge, $Q$ & C [As] & Displacement, $x$ & $\mathrm{~m}$ \\
\hline Acoustic compliance, $C_{a}$ & $\mathrm{~m}^{3} / \mathrm{Pa}=\mathrm{m}^{5} / \mathrm{N}$ & Capacitance, $C_{e}$ & $\mathrm{~F}$ & Compliance, $C_{m}$ & $\mathrm{~m} / \mathrm{N}$ \\
\hline Acoustic stiffness $K_{a}$ & $\mathrm{~Pa} / \mathrm{m}^{3}=\mathrm{N} / \mathrm{m}^{5}$ & Capacitance & $\mathrm{F}^{-1}$ & Stiffness & $\mathrm{N} / \mathrm{m}$ \\
\hline Acoustic mass, $M_{a}$ & $\mathrm{~kg} / \mathrm{m}^{4}=\mathrm{Ns}^{2} / \mathrm{m}^{5}$ & Inductance, $L_{e}$ & $H$ & Mass, $M_{m}$ & $\mathrm{~kg}$ \\
\hline Acoustic resistance, $R_{a}$ & $\mathrm{~Pa} \mathrm{~s} / \mathrm{m}^{3}=\mathrm{Ns} / \mathrm{m}^{5}$ & Resistance, $R_{e}$ & $\Omega$ & Resistance, $R_{m}$ & $\mathrm{Ns} / \mathrm{m}$ \\
\hline
\end{tabular}

section, the different interfered domains in the micro-sensor function will be analysed and discussed in order to derive the final equivalent lumped model.

\subsection{Equivalent Electro-Acoustic Circuit of the Micro-Sensor}

The sensitivity depended on the domains involved in the micro-sensor operation principle (acoustic-mechanicalmagnetic). Initially, in the acoustic domain when the pressure hit the diaphragm surface, an acoustic wave radiated outward. This could be modelled using radiation impedance composed of an acoustic resistance and mass expressed as: ${ }^{24}$

$$
\underline{Z_{r a d}^{a c}}=R_{\text {rad }}+j \omega M_{\text {rad }}=\frac{1}{8} \frac{\rho_{a i r}}{c_{a i r}} \omega^{2}-j \frac{4}{3 \pi} \frac{\rho_{a i r}}{L} \omega ;
$$

where $j$ denoted the imaginary unit, $\rho_{\text {air }}$ was the air density and $c_{a i r}$ was the sound velocity in the air. On the other hand, when the incident pressure $P_{\text {in }}$ physically hit the diaphragm top surface, this latter fluctuated. Therefore, its behaviour could be modelled by a mechanical stiffness and mass expressed, respectively, by Eq. (6) and Eq. (7). When the effect of the axial stress appearing during fabrication could be neglected, this gave:

$$
\underline{Z_{m e m}^{m e}}=j \omega M_{d i a}^{m e c}+\frac{K_{d i a}^{m e c}}{j \omega} .
$$

The diaphragm fluctuation was due to the acoustic pressure that caused a pressure variation in the gap underneath the diaphragm, $P_{\text {cav }}$ (Fig. 5). Since the volume of the air in the micromachined cavity underneath the diaphragm, closed at its end, was compressible, it could be modelled as an acoustic compliance. Assuming that no significant flow was present in the rear cavity and that the air was considered as a perfect gas, the acoustic impedance of the cavity could be assimilated by:

$$
\underline{Z_{b a c k}^{a c}}=\frac{\rho_{a i r} c_{a i r}^{2}}{j \omega V}=\frac{K_{c a v}^{a c}}{j \omega} ;
$$

where $V$ was the volume of the back cavity, whose equivalent mass and losses were neglected in our model. This assumption was valid if the cavity side length was bounded between $0.05 \times \sqrt{(\pi / f)}$ and $(10 \times \sqrt{\pi)} / f(f$ was the possible incident wave frequency). In the used micro-sensor attachment structure, we needed to add a small vent to equalize the atmospheric and back cavity pressures.

A slot (with length $\alpha$, width $w_{h}$ and thickness $l_{h}$ ) was considered narrow if its width (or diameter) was less than
$0.003 / \sqrt{f}$. The passage of sound waves in a narrow slot was influenced by the acoustic resistance that was due to the viscosity of the air. In this case, the flow of air, supposed to be incompressible, through the capillary slot could be described by acoustic impedance, described by an acoustic resistance in series with a mass: ${ }^{22}$

$$
\underline{Z_{\text {hole }}^{a c}}=R_{\text {hole }}^{a c}+j \omega M_{\text {hole }}^{a c}=\frac{12 \eta_{\text {air }} l_{h}}{\alpha^{3} w_{h}}+j \omega \frac{12 \rho_{\text {air }} l_{h}}{\alpha w_{h}} ;
$$

where $\eta_{a i r}$ was the dynamic viscosity of the air. Finally, the diaphragm displacement generated an induced voltage at the ends of the inner inductor. Therefore, a last electro-magnetic phenomenon was considered and included, which was the electromagnetic induction link. This produced an electromotive force according to Faraday's law of induction.

All of the above introduced parameters could be gathered in a single lumped elements model representing the previously explained effects (see Fig. 5). The developed circuit related the different domains through transformers and/or gyrators with appropriate coupling coefficients. The coupling coefficient between mechanical and acoustic domains was $S=L_{2}$, which represented the diaphragm surface. In fact, this coefficient related also the flow rate $w$ and the velocity of the diaphragm $v$ as:

$$
\left\{\begin{array}{l}
F=S P \\
w=S v
\end{array} ;\right.
$$

where $F$ was the mechanical force acting on the diaphragm. On another side, the fluctuation velocity of the diaphragm was related to the electromotive force, $e$, through a gyrator, as shown in Fig. 6, via this couple of equations:

$$
\left\{\begin{array}{c}
F_{\text {Lorentz }}=K_{z} i_{2} \\
e=K_{z} v
\end{array} .\right.
$$

The coefficient $K_{z}$ was defined in the previous work as the coupling gain between the mechanical displacement and the electromotive force, and was given by: ${ }^{17}$

$$
e=A_{2} I_{1} \xi v=K_{z} v ;
$$

where $\xi$ was the out-of-plane diaphragm displacement which was a frequency dependent, $I_{1}$ was the current flowing in the outer inductor and $A_{2}$ was a geometric constant depending on the average distance between the two inductors $\varepsilon_{a}$ and the coil side $a$, and were given by: ${ }^{17}$ 


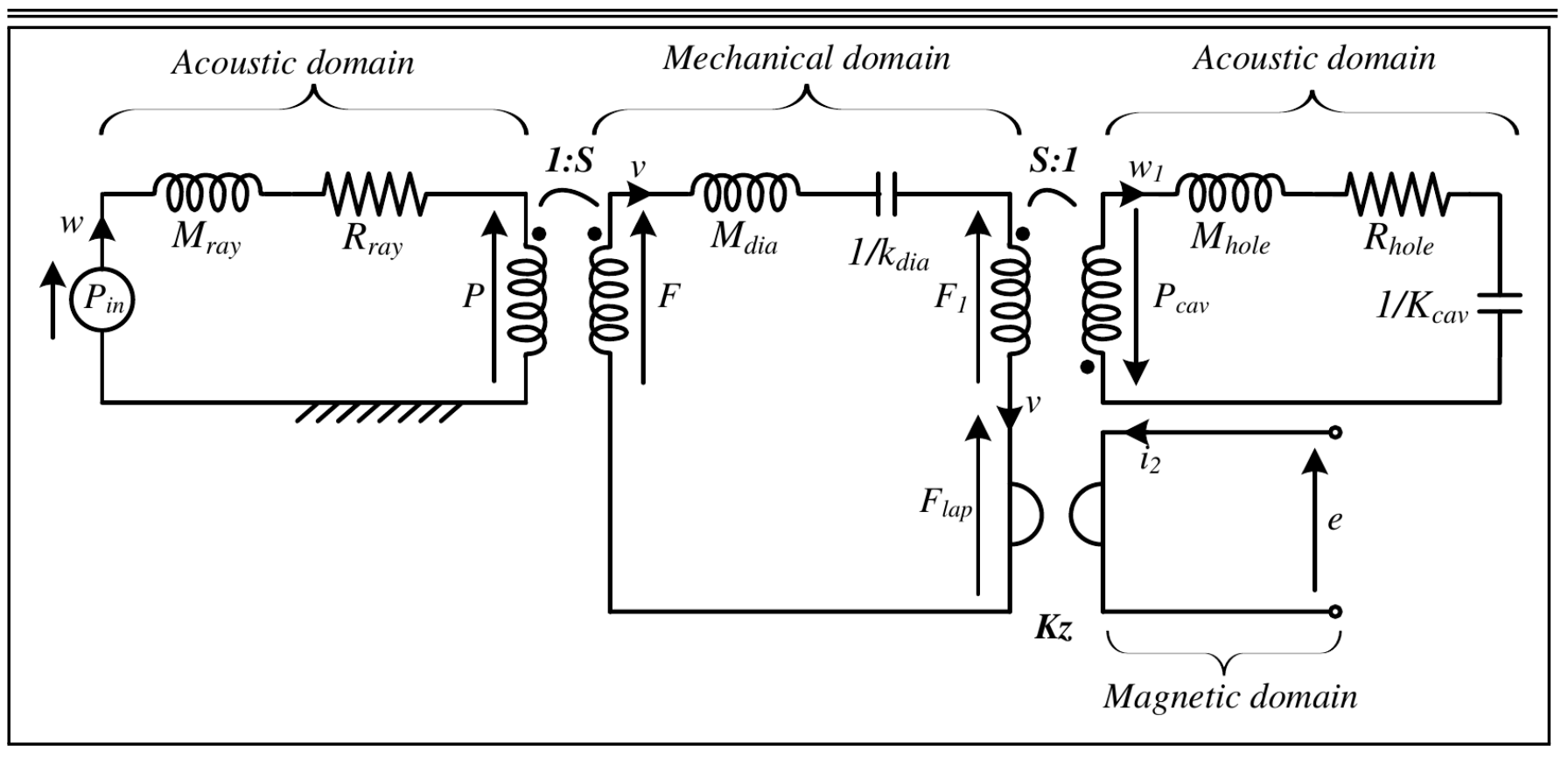

Figure 5. Lumped element model showing the coupled domains in the micro-sensor.

$$
\begin{aligned}
w & =\frac{S^{2} P_{\text {in }} p}{p^{2}\left[S^{2}\left(M_{\text {ray }}^{a c}+M_{\text {hole }}^{a c}\right)+M_{\text {dia }}^{m e c}\right]+p S^{2}\left[R_{\text {ray }}^{a c}+R_{\text {hole }}^{a c}\right]+\left[K_{\text {dia }}^{m e c}+S^{2} K_{c a v}^{a c}\right]} \\
\frac{e}{P_{\text {in }}} & =\frac{S K_{z} p}{p^{2}\left[S^{2}\left(M_{\text {ray }}^{a c}+M_{\text {hole }}^{a c}\right)+M_{\text {dia }}^{m e c}\right]+p S^{2}\left[R_{\text {ray }}^{a c}+R_{\text {hole }}^{a c}\right]+\left[K_{\text {dia }}^{m e c}+S^{2} K_{\text {cav }}^{a c}\right]}
\end{aligned}
$$

$$
A_{2}=\frac{2 \mu_{0} n_{1} n_{2}}{\pi \varepsilon_{a}^{2}}\left(\sqrt{\varepsilon_{a}^{2}+\left(a-\varepsilon_{a}\right)^{2}}-\sqrt{2} \varepsilon_{a}\right)
$$

where $n_{1}$ and $n_{2}$ were the numbers of turns of the inner and outer inductors, respectively, and $\mu_{0}$ was their magnetic constant. So, $K_{z}$ coefficient related the force $F_{\text {Lorent } z}$ to the induced current $i_{2}$ in the inner inductor, and related the induced voltage with the mechanical velocity of the diaphragm. The mechanical-magnetic link was modelled by a gyrator, unlike the acousto-mechanical link, which was modelled by a transformer. An ideal electrical gyrator related the input voltage to the output current and the input current to the output voltage, which meant in our case, that the Lorentz force on one port was coupled to the current on the other port, and the induced voltage was coupled to the diaphragm velocity (see Fig. 5).

In order to simplify the micro-sensor lumped component model, we used standard relations between parameters on each side of transformers and the gyrator. Generally, the mechanical part was represented by a force $F$ and an impedance $Z^{m}$, while the acoustic part, by an acoustic pressure $p$ and an impedance $Z^{a}$, as shown in Fig. 6(a). In Fig. 6(b), the acoustic equivalent circuit without transformer is represented, where the primary was brought to the secondary. It contained the acoustic equivalent impedances and source given by $Z^{a m}=Z^{m} / S^{2}$ and $p_{F}=F / S$. The contrary was done for the mechanical equivalent circuit, where the acoustic part was brought to the mechanical part (see Fig. 6(c)).

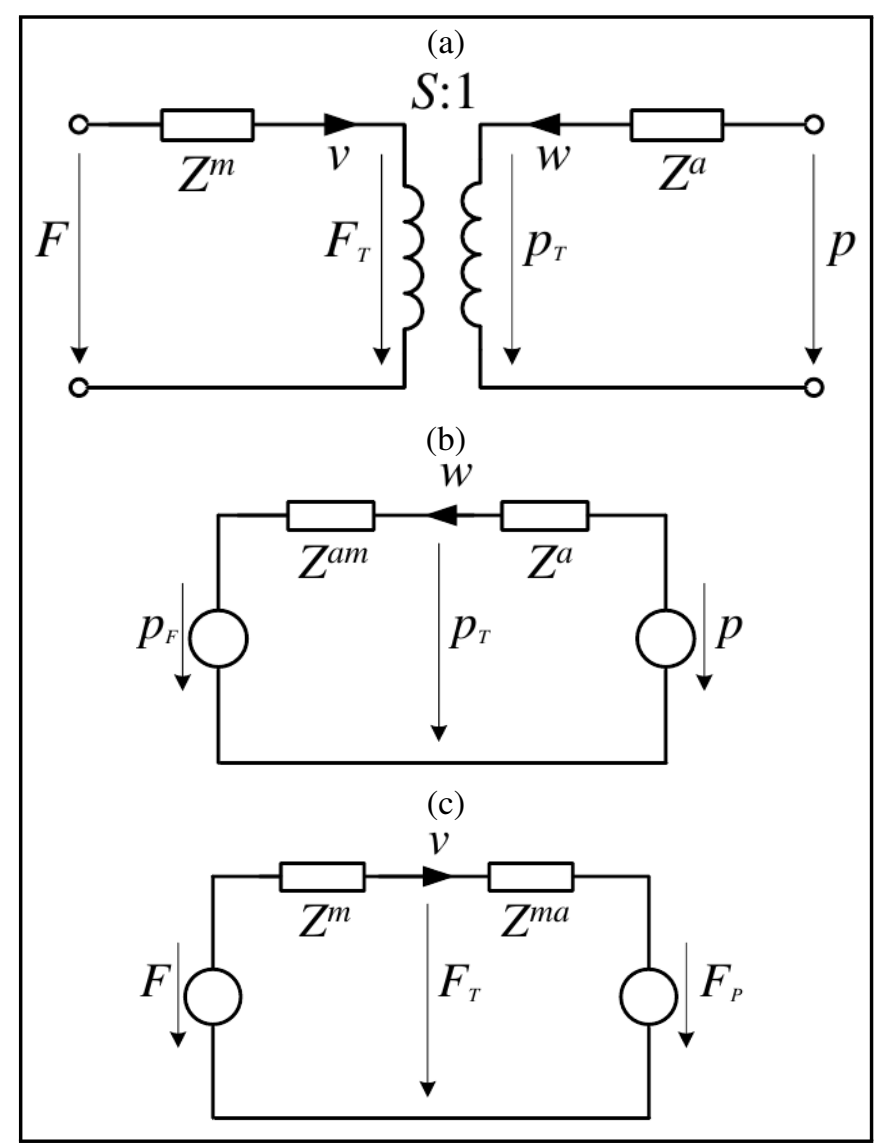

Figure 6. Representation of the mechanical-acoustic coupling in the form of (a) coupling scheme (b) equivalent acoustic scheme (c) equivalent mechanical scheme. 


\subsection{Frequency Response and Sensitivity}

After simplifying the circuits by bringing all the components in the acoustic domain, and using the Kirchhoff's laws, the acoustic flow could be expressed by Eq. (15), where $p$ was the Laplace variable $(p=j \omega)$. In our model, we assumed that the force $F$, due to the incident pressure, was high compared to the electromagnetic force $F_{\text {Lorent } z} .{ }^{28}$ Finally, when using Eq. (19) and Eq. (15), the relation between the induced voltage and the incident pressure could be given by Eq. (16).

From Eq. (16), we noticed that the transfer function between the induced voltage and the applied pressure, and thus the overall sensitivity of the micro-sensor (defined by $\left.S_{e n}=\Delta e / \Delta P_{i n}\right)$, increased by increasing the coefficient $K_{z}$, which was proportional to the increase of the current, and the diaphragm displacement. So, for a fixed diaphragm side length of $1.5 \mathrm{~mm}$, sensitivity values are plotted in Fig. 7 as a function of frequency for different diaphragm thicknesses. We observed that for the three structures, the sensitivity had a narrow bandwidth with a maximum situated at the diaphragm resonant frequency. From the same figure, we also deduced that the sensitivity decreased as diaphragm thickness increased which in turn gave a stiffer suspended structure, resulting in a sensitivity drop. For the three used thicknesses, the corresponding sensitivity values were $2.23 \mathrm{mV} / \mathrm{Pa}, 0.28 \mathrm{mV} / \mathrm{Pa}$ and $6 \mu \mathrm{V} / \mathrm{Pa}$ (with a bandwidth of few Hertz). We concluded that the developed micro-sensor design behaved as a mono-frequency detector. It could be used to induce a high sensitivity only around the already specific frequency, with a narrow bandwidth. This result must be taken into consideration when setting the application features, in which the inductive transducer will be used. The obtained sensitivity shape was due to the dependence of Eq. (16) on the diaphragm displacement. This displacement originated from the radial magnetic field, which was as well linearly proportional to $\xi$, for low amplitude fluctuation value. The adjustment of some acoustic parameters, as cavity stiffness and mass of the capillary slot, was mandatory in order to tune the resonant frequency, which can shift from its original position.

In Fig. 8, we show the variation of the sensitivity peak value on the resonant frequency, depending on the diaphragm thickness. To set a high resonant frequency, we needed to increase the thickness on the detriment of decreasing sensitivity. The sensitivity of the micro-sensor could drop from $2.23 \mathrm{mV}$ to around $6 \mu \mathrm{V} / \mathrm{Pa}$ when the resonance frequency was set around $47 \mathrm{kHz}$. The obtained sensitivity performances made the design very useful in the applications, which required a high sensitivity within a narrow bandwidth (resonance model). ${ }^{29,30}$ Examples included acoustic emission sensors and an ultrasonic microphone to detect partial discharge measurement (with center frequency of $40 \mathrm{kHz}$ ). ${ }^{31,32}$ Better sensitivity around the mechanical resonance frequency resulted from the electrodynamic micro-sensor, when used without a damping mechanism. It could be three orders of magnitude higher in presence of damping. ${ }^{21}$ On the other hand, increasing the system damping did not affect/change the sensitivity shape, which remained narrow, but rather it decreased the sensitivity values

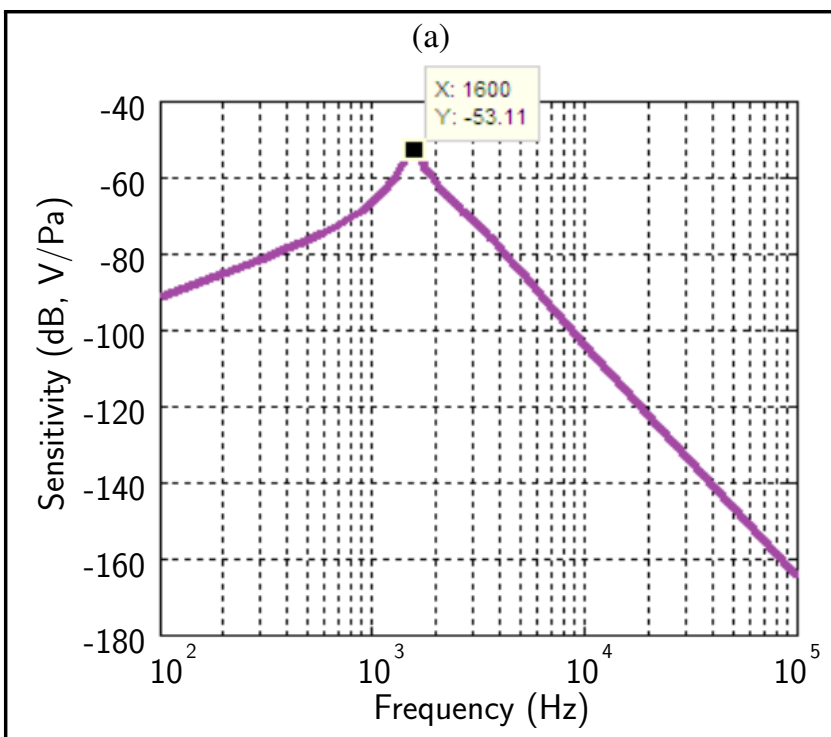

(b)

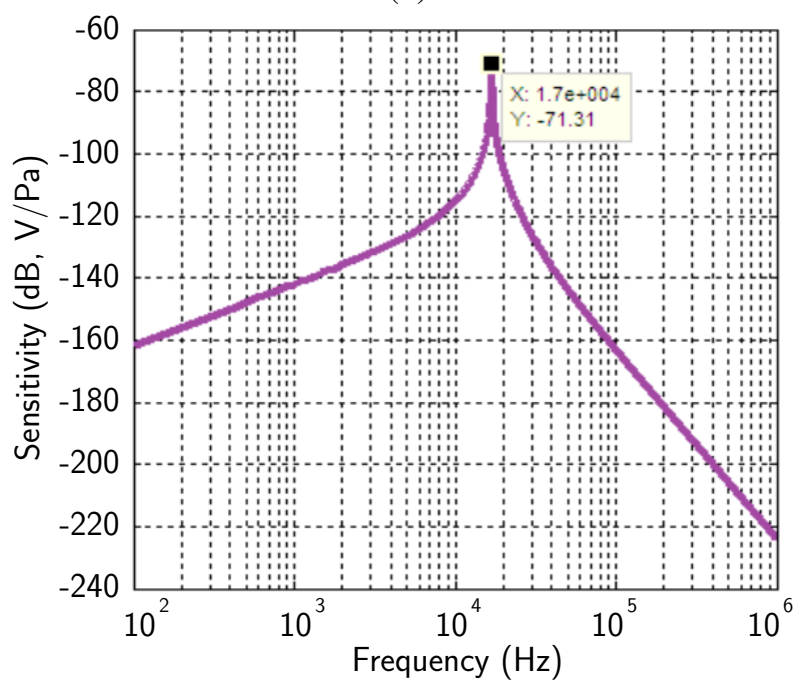

(c)

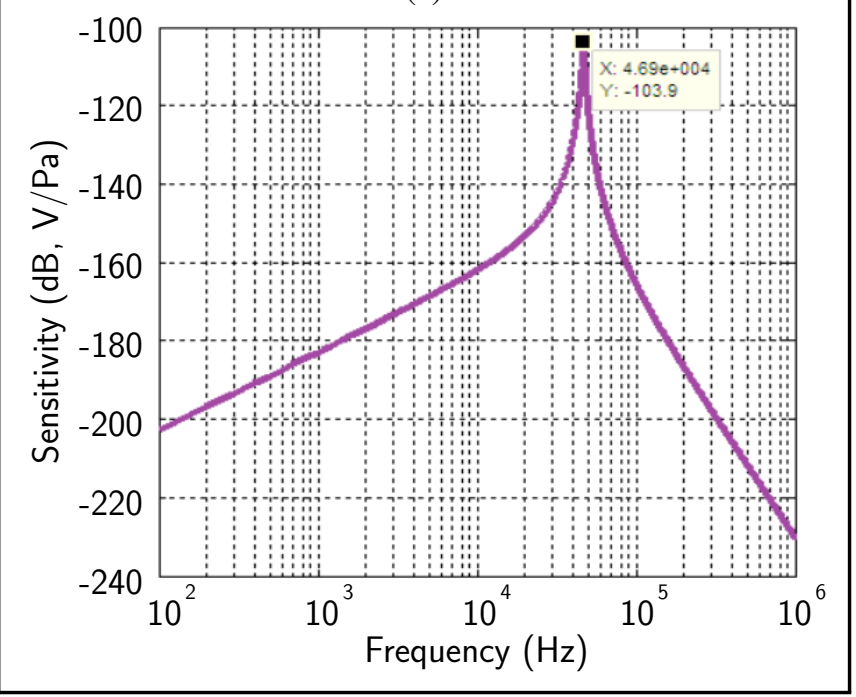

Figure 7. Evaluated electrodynamic micro-sensor sensitivity as a function of frequency for a diaphragm length of $1500 \mu \mathrm{m}$ and thicknesses $t_{h}$ of (a) $0.3 \mu \mathrm{m}$, (b) $3 \mu \mathrm{m}$, and (c) $10 \mu \mathrm{m}$. 


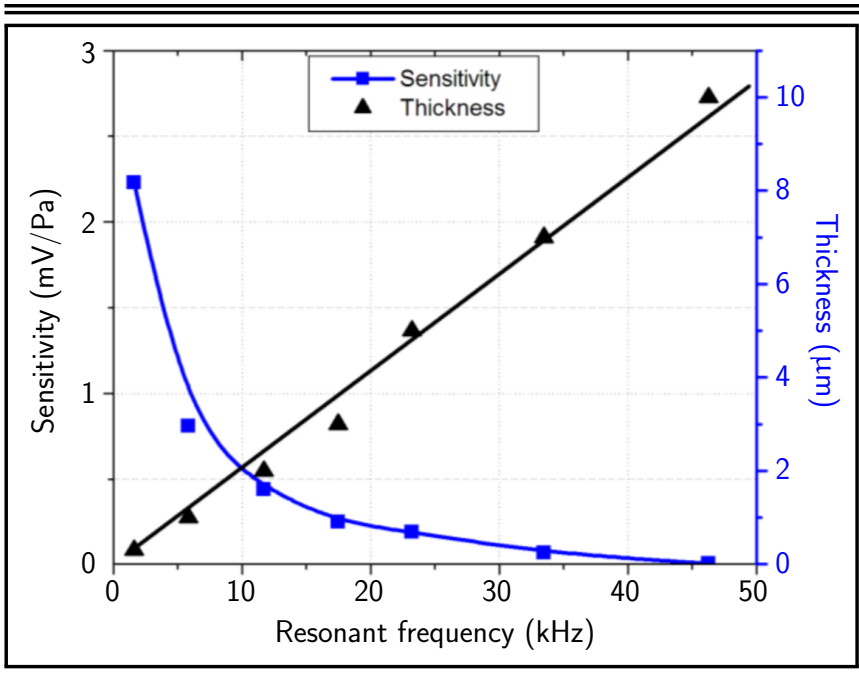

Figure 8. Sensitivity and thicknesses vs resonant frequency for a diaphragm side length of $1500 \mu \mathrm{m}$.

Table 6. Main parameters used for sensitivity evaluation of the micro-sensor.

\begin{tabular}{||c|c|}
\hline Parameter & Value \\
\hline Number of turns, $n_{1}$ and $n_{2}$ & 50 turns \\
\hline Distance between both inductor centers $\left(\varepsilon_{a}\right)$ & $324 \mu \mathrm{m}$ \\
\hline Outer inductor average length $(a)$ & $1604 \mu \mathrm{m}$ \\
\hline Inductor width $(w)$ and thickness $\left(t_{i}\right)$ & $1 \mu \mathrm{m}$ \\
\hline Pitch between spirals $(s)$ & $1 \mu \mathrm{m}$ \\
\hline Diaphragm side $(L)$ & $1500 \mu \mathrm{m}$ \\
\hline Gap thickness $($ substrate thickness) $(h)$ & $675 \mu \mathrm{m}$ \\
\hline Hole length $\left(l_{h}\right)$ & $200 \mu \mathrm{m}$ \\
\hline Hole width $\left(w_{h}\right)$ and side $(b)$ & $50 \mu \mathrm{m}$ \\
\hline
\end{tabular}

when the fluctuation amplitude decreased. However, enlarging the micro-sensor bandwidth required achieving a design, which was based on diaphragm velocity conversion, which was different compared to our case, depending on diaphragm displacement conversion. Nevertheless, enlarging the bandwidth significantly reduced the overall sensitivity and vice versa. The final dimensions and various parameters used for our model are shown in Table 6. The next steps concerning this study will focus on layout drawing of the device followed by an experimental characterization and validation.

\section{CONCLUSIONS}

In this paper, we perform both modal and harmonic modelling for an electrodynamic MEMS micro-sensor of dynamic pressure over a large bandwidth. Firstly, the resonant frequency is deduced for different possible diaphragm thicknesses, using both analytic and FEM simulation. Thereafter, the static and harmonic displacements are deduced showing an optimum around the chosen resonant frequency of the diaphragm. Finally, the dynamic sensitivity of the micro-sensor is found by gathering all parameters from involved domains. We can conclude that the adopted electrodynamic transducer design gives a relatively high sensitivity around the resonant frequency but within a narrow bandwidth. This result is due to the dependence of the sensitivity on the diaphragm displacement originating from electromagnetic coupling coefficient that is linearly proportional to the diaphragm displace- ment. The micro-sensor sensitivity is around few $\mathrm{mV} / \mathrm{Pa}$ for small resonant frequencies and drops down to the $\mu \mathrm{V} / \mathrm{Pa}$ range for ultrasonic frequencies.

\section{ACKNOWLEDGEMENTS}

The authors acknowledge the support for the Article Processing Charge by the Tunisian Ministry of Higher Education and Scientific Research through the Programme des Jeunes Enseignants Chercheurs (PJEC) in the field of Engineers Science (Grant Ref: 18PJEC12-16).

\section{REFERENCES}

1 Minhang, B. Analysis and design principles of MEMS devices, Elsevier, Amsterdam, The Netherlands, 104-105, (2005).

2 Roessig, T. A., Howe, R. T., Pisano, A. P., and Smith, J. H. Surface micromachining resonant accelerometer, International conference on solid state Sensors and Actuators, Chicago, Illinois, USA, (1997). https://dx.doi.org/10.1109/SENSOR.1997.635237

3 Oh, Y., Lee, B., Baek, S., Kim, H., Kim, J., Kang, S., and Song, C. Surface- micromachined tunable vibratory gyroscope, IEEE International Micro-electromechanical Systems Workshop, Nagoya, Japan, (1997). https://dx.doi.org/10.1109/MEMSYS.1997.581824

${ }^{4}$ Said, M. H., Tounsi, F., Gkotsis, P.,Mezghani, B., and Francis, L. A. A resonant microstructure tunability analysis for an out-of-plane capacitive detection MEMS magnetometer, Springer Journal of Microsystem Technologies, 23 (7), 2599-2608, (2017). https://dx.doi.org/10.1007/s00542016-3093-y

5 Magori, V., and Walker, H. Ultrasonic presence sensors with wide range and high local resolution, ultrasonics, ferroelectrics and frequency control, IEEE Transactions on Ultrasonics Ferroelectrics and Frequency Control, 34 (2), 202-211, (1987). https://dx.doi.org/10.1109/TUFFC.1987.26933

6 Neumann Jr, J. J., and Gabriel, K. J. CMOSMEMS membrane for audio-frequency acoustic actuation, Sensors and Actuators A, 95 (2-3), 175-182, (2002). https://dx.doi.org/10.1016/S0924-4247(01)00728-2

7 Ganji, B. A., and Majlis, B. Y. Design and fabrication of a new MEMS capacitive microphone using a perforated aluminum diaphragm, Journal of Sensors and Actuators A, 149 (1), 29-37, (2009). https://dx.doi.org/10.1016/j.sna.2008.09.017

8 Sugandi, G., and Majlis, B. Y. Fabrication of MEMS based microspeaker using bulk micromachining technique, Advanced Materials Research, 254, 171-174, (2011). https://dx.doi.org/10.4028/www.scientific.net/AMR.254.171 
${ }^{9}$ Mattila, T., Kiihamäki, J., Lamminmäki, T., Jaakkola, O., Rantakari, P., Oja, A., Seppä, H., Kattelus, H., and Tittonen, I. A $12 \mathrm{MHz}$ micromechanical bulk acoustic mode oscillator, Sensors and Actuators A, 101 (1-2), 1-9, (2002). https://dx.doi.org/10.1016/S0924-4247(02)00204-2

10 Zaki, A., Elsimary, H., and Zaghloul, M. Miniature SAW device using MEMS technology, Microelectronics Journal, 38 (3), 426-429, (2007). https://dx.doi.org/10.1016/j.mejo.2006.11.010

11 Horowitz, S., Nishida, T., Cattafesta, L., and Sheplak, M. Development of a micromachined piezoelectric microphone for aeroacoustics applications, Journal of Acoustical Society of America, 122 (6), 3428-3436, (2007). https://dx.doi.org/10.1121/1.2785040

12 Arnold, D. P., Gururaj, S., Bhardwaj, S., Nishida, T., and Sheplak, M. A piezoresistive microphone for aeroacoustic measurements, International Mechanical Engineering Congress and Exposition, New York, New York, USA, (2001)

${ }^{13}$ Hansen, S. T., Ergun, A. S., Liou, W., Auld, B. A., and Khuri-Yakub, B. T. Wideband micromachined capacitive microphones with radio frequency detection, The Journal of the Acoustical Society of America, 116 (2), 828-842, (2004). https://dx.doi.org/10.1121/1.1771617

${ }^{14}$ Horng, R. -H., Chen, K. -F., Tsai, Y. -C., Suen, C. Y., and Chang, C. -C. Fabrication of a dual-planar-coil dynamic microphone by MEMStechniques, Journal of Micromechanical and Microengineering, 20, 1-7, (2010). https://dx.doi.org/10.1088/0960-1317/20/6/065004

15 Bakhoum, E. G. Micro-and nano-scale sensors and transducers, Chapter 1, CRC Press, Boca Raton, Florida, (2015).

16 Arshad, A., and Tasnim, R. An inductive transducer based pressure sensor for biomedical applications, IEEE International Conference on Informatics, Electronics \& Vision (ICIEV), Fukuoka, Japan, (2015). https://dx.doi.org/10.1109/ICIEV.2015.7333987

17 Tounsi, F., Said, M. H., Rufer, L., Mezghani, B., and Masmoudi, M. Optimization of induced voltage from CMOScompatible MEMS electrodynamic microphone with coaxial planar, IEEE Sensors Journal, 16 (18), 6879-6889, (2016). https://dx.doi.org/10.1109/JSEN.2016.2589271

${ }^{18}$ Polak, F., Sikorski, W., and Siodła, K. Prototype measurement system for localization of partial discharges sources - microphone array, Computer Applications in Electrical Engineering, 13, 209-219, (2015).

19 Nanoelectronics centre in IIT-Mumbai India, retrieved from http://www.cen.iitb.ac.in/cen/index.php, (Accessed February 16,2018$)$.

20 Said, M. H., Sandeep, G. S., Tounsi, F., Mezghani, B., Masmoudi, M., and Rao, V. R. Numerical magnetic analysis for a monolithic micromachined electrodynamic microphone, ICMEMS Conference, IIT MADRAS-India, (2014).

${ }^{21}$ Hadj Said, M., Tounsi, F., Surya, S. G., Mezghani, B., Masmoudi, M., and Rao, V. R. A MEMS-based shifted membrane electrodynamic microsensor for microphone applications, Journal of Vibration and Control, 24 (1), 208-222, (2018). https://dx.doi.org/10.1177/1077546316637298

${ }^{22}$ Esteves, J., Rufer, L., Basrour, S., and Ekeom, D. CMOSMEMS technology with front-end surface etching of sacrificial $\mathrm{SiO}_{2}$ dedicated for acoustic devices, 5th IEEE International Workshop on Advances in Sensors and Interfaces (IWASI'13), Bari, Italy, (2013).

${ }^{23}$ Zhou, Z., Rufer, L., and Wong, M. Damped aero-acoustic microphone with improved high-frequency characteristics, IEEE Microelectromechanical Systems, 23 (5), 1094-1100, (2014). https://dx.doi.org/10.1109/JMEMS.2014.2304730

24 Tounsi, F. Microphone électrodynamique MEMS en technologie CMOS, étude, modélisation et réalisation, $P h D$ Thesis Dissertation, Grenoble Alpes University, Grenoble, France, Chapter 3, 100, (2010).

25 Dominguez, C. C. Conception de transducteurs acoustiques micro-usiné, PhD Thesis Dissertation, Grenoble Alpes University, Grenoble, France, Chapter 3, 67, (2005).

${ }^{26}$ Sampaio, R. Microelectromechanical systems (MEMS) for applications in acoustics, Master's Thesis, Instituto Superior Tecnico, Lisbon, Portugal, Chapter 3, 55, (2013).

${ }^{27}$ Carr, A. J. Dynamic analysis of structures, Bulletin of New Zealand society for earthquake engineering, 27 (2), (1994).

28 Bruneau, M. Manuel d'acoustique fondamentale, Hermès Science Publications, 576, (1998), ISBN: 2866017129.

${ }^{29}$ Huang, S., Li, M., Xu, Y., Xu, D., Xie, X., and Cheng, $X$. Research on embedded sensors for concrete health monitoring based on ultrasonic testing, 4thInternational Conference on the Durability of Concrete Structures, Purdue University, West Lafayette, Indiana, (2014). https://dx.doi.org/10.5703/1288284315429

${ }^{30}$ Posada-Roman, J., Garcia-Souto, J. A., and RubioSerrano, J. Fiber Optic sensor for acoustic detection of partial discharges in oil-paper insulated electrical systems, Sensors, 12, 4793-4802, (2012). https://dx.doi.org/10.3390/s120404793

${ }^{31}$ Greve, D. W., Oppenheim, I. J., Wrightb, A. P., Wu, W., and Wrigh, A. P. Design and testing of a MEMS acousticemission sensor system, Proceedings of SPIE - The International Society for Optical Engineering, 6529 (12), 1-9, (2008). https://dx.doi.org/10.1117/12.775777

32 Van Haeren, R., Stone, G. C., Meehan, J., and Kurtz, M. Preventing failures in outdoor distribution class metalclad switchgear, IEEE Transactions on Power Apparatus and Systems, 104 (10), 2706-2712, (1985). https://dx.doi.org/10.1109/TPAS.1985.319111 